\title{
Cell-free expression and functional reconstitution of CALM in clathrin assembly
}

\author{
Jeong-Ah Kim ${ }^{1}$ and Hyung-Lae Kim ${ }^{1,2}$ \\ ${ }^{1}$ Department of Biochemistry, Medical College, Ewha Womans Uni- \\ versity, Seoul 158-710, Korea \\ ${ }^{2}$ Corresponding author: Tel, +82-2-650-5727; \\ Fax, +82-2-650-5791; E-mail, hyung@mm.ewha.ac.kr
}

Accepted 23 June, 2001

Abbreviations: CCV; clathrin-coated vesicle, CALM; clathrin assembly protein lymphoid myeloid, GST; glutathion sulfur transferase, SH3; src homology 3

\begin{abstract}
Clathrin-mediated vesicle formation is an essential step in the intracellular trafficking of the protein and lipid. Binding of clathrin assembly protein to clathrin triskelia induces their assembly into clathrin-coated vesicles (CCVs). In order to better understand a possible role of post-translational modification of CALM (clathrin assembly protein lymphoid myeloid), the homologue of AP180, in the assembly of CCVs, CALM was expressed in the cell-free reticulocyte translation system that is capable of carrying out post-translational modification. The apparent molecular weight of the expressed recombinant CALM was estimated as $105 \mathrm{kD}$. Alkaline phosphatase treatment of CALM resulted in a mobility shift on SDSPAGE. We found that CALM was associated with the proteins harboring $\mathrm{SH} 3$ domain, promote assembly of clathrin triskelia into clathrin cage and bound to the preformed clathrin cage. CALM was also proteolyzed by caspase 3 and calpain but not by caspase 8. These results indicated that the post-translationally modified CALM, expressed in the eukaryotic cell-free reticulocyte translation system was able to mediate the assembly of clathrin and the coatedvesicle formation.
\end{abstract}

Keywords: expression, clathrin-coated vesicle, CALM, $\mathrm{SH} 3$ domain, cleavage

\section{Introduction}

Clathrin-mediated vesicle formation is an essential step in the intracellular trafficking of the membrane compart- ment. Most of the transport vesicles arise from the specialized coated regions of the membrane and bud off as coated vesicles with distinctive vesicle contents (Alberts et al., 1994). Clathrin, the most abundant component of coat - forming proteins makes a highly ordered structure on the cytoplasmic surface of the vesicle. Soluble clathrin $(\sim 650 \mathrm{kD})$ is composed of identical three $160 \mathrm{kD}$ heavy chains and three 22-28 kD light chains. These three heavy chains and three light chains form three lagged trimer complex, called clathrin triskelion. This triskelion assembles into a basketlike framework of hexagons and pentagons to form coated pits on the cytoplasmic surface of membrane (Marsh et al., 1999). The second major coat proteins are assembly proteins (assembly or adaptor proteins, AP).

Clathrin assembly proteins belong to two gene family, the tetrameric or monomeric AP family. Four tetrameric APs have been described and designated AP-1 (Ahle et al., 1986), AP-2 (Beck et al., 1992), AP-3 (Faundez et al., 1998), and AP-4 (Dell'Angelica et al., 1999). AP-1 and AP-2 were first characterized as the major clathrin coated vesicle adaptor proteins. AP-1 was found to localize at clathrin coated vesicles budding from Golgi membrane and AP-2 at clathrin-coated vesicles budding from plasma membrane, but AP-3 has only recently been identified and appeared to be associated with clathrin coated protein as well (Faundez et al., 1998). AP-3 plays a role in trafficking from trans-Golgi network to the lysosome (Simpson et al., 1997). AP-4 has also been identified but not been characterized in detail.

Monomeric APs include AP180 and CALM. AP180 was mainly found to be present at synapse (Stephan et al., 1990), while CALM is expressed in most of all tissues (Dreyling et al., 1996; Kim et al., 1999). The native AP180 was shown to interact with clathrin triskelia and thereby to induce clathrin assembly through C-terminal domain (Ye et al., 1995). AP180 is a glycosylated and phosphoorylated acidic protein (Keen et al., 1986; Bar-Zvi et al., 1988; Murphy et al., 1991; Morris et al., 1993). AP180 contains high affinity binding sites for inositides, the binding of which inhibits their ability to promote clathrin assembly (Norris et al., 1995).

Recently, the CALM, which is homologous to AP180, was reported in human and rat tissue (Dreyling et al., 1995; Kim et al., 1999). Bacterially expressed CALM was able to induce the assembly of clathrin cage and bind to clathrin triskelia, like AP180 through C terminal domain. GST-CALM could interact with $\mathrm{SH} 3$ domain, and be proteolysed by calpain and caspase (Kim et al., $2000 \mathrm{~b})$. The CALM was found to be colocalized with 
AP-2 and clathrin heavy chain in plasma membrane and coated pits (Tebar et al., 1999). However, whether contribution of any post-translational modification of CALM, especially the cellular signal mediated phosphorylation would affect the clathrin-mediated vesicle formation is not clearly understood. For further elucidation of the interrelationship between the structural modification of CALM and the protein-protein interaction during vesicle formation, CALM was expressed using the cell-free reticulocyte lysate translation system. The results showed the glycosylated and phosporylated CALM retained its functional properties as AP in clathrin vesicle formation.

\section{Materials and Methods}

\section{Materials}

All buffers used in the protein work contained $0.1 \mathrm{mM}$ PMSF (phenylmethylsulfonylfluoride). Protein concentrations were determined by BCA kit according to the protocol by manufacturer's instruction (Pierce). The recombinant caspase 3 and 8 were the generous gifts from Dr. Y. G. Chung (Kwang-Ju KAIST, Kwang-Ju, Korea). Plasmid expressing $\mathrm{SH} 3$ domain was provided by Dr S. R. Kim (Chungbuk Univ. Cheongju, Korea)

\section{Preperation of clathrin}

All procedures were performed at $4^{\circ} \mathrm{C}$. Fresh bovine brains were chopped and minced after stripping out the meninges. And then it was homogenized using the equal volume of homogenizing buffer in a Waring blender. The homogenizing buffer consisted of $0.1 \mathrm{M}$ 2-(N-morpholino) ethansulfonic acid (MES), $1 \mathrm{mM}$ EGTA, $0.5 \mathrm{mM}$ $\mathrm{MgCl}_{2}$, and $0.02 \% \mathrm{NaN}_{3}, \mathrm{pH}$ 6.5. The homogenate was centrifuged at $19,000 \mathrm{~g}$ in a Sorval GSA rotor for $40 \mathrm{~min}$. The supernatant was saved and centrifuged at $43,000 \mathrm{~g}$ in a Beckman $45 \mathrm{Ti}$ rotor for $1 \mathrm{~h}$. The pellet containing the coated vesicles was resuspended in homogenizing buffer and gently homogenized using Dounce glass homogenizer. The resulting pellet is the crude coatedvesicle. To extract clathrin and associated proteins, the crude coated-vesicle was resuspended in the extration buffer, which composed of $0.5 \mathrm{M}$ Tris, $\mathrm{pH} 7.0,2 \mathrm{mM}$ dithiothreitol (DTT), $1 \mathrm{mM}$ EDTA, and homogenized using Dounce glass homogenizer. The homogenate was stored at $4^{\circ} \mathrm{C}$ overnight. The extract was centrifuged at 43,000 $g$ for $4 \mathrm{~h}$, and the supernatant was applied to the sepharose CL-4B column $(2 \times 100 \mathrm{~cm})$ which was equilibrated with the extraction buffer. The column was eluted with the equilibration buffer, and the first major peak was used as the clathrin sample.

\section{In vitro translation}

Proteins were expressed using in vitro translation kit containing TNT T7 coupled reticulocyte lysate system (Promega) by manufacturer's instruction. The plasmid encoding the full length cDNA of CALM (Kim et al., 1999) was used for the in vitro transcription/translation system containing translation grade $\left[{ }^{35} \mathrm{~S}\right]$ methionine (1,000 Ci/mmol, Amersham Pharmacia).

\section{Clathrin cage binding assay}

Clathrin triskelia $(1.8 \mu \mathrm{M})$ was dialysed overnight in isolation buffer (0.1 M MES, pH 6.7, $1 \mathrm{mM}$ EGTA, 0.5 $\mathrm{mM} \mathrm{MgCl}, 0.1 \mathrm{mM}$ PMSF) with $3 \mathrm{mM} \mathrm{CaCl}_{2}$ at $4^{\circ} \mathrm{C}$. Newly assembled cages were precipitated by ultracentrifugation $(100,000 \mathrm{~g})$ in a Beckman TLA 100.3 rotor. The pellet was suspended in isolation buffer, and incubated with assembly protein $\left(\left[{ }^{35} \mathrm{~S}\right]\right.$-methionine labeled CALM protein, reticulocyte lysate) for $1 \mathrm{~h}$ on ice. The mixtures were ultracentrifuged for $20 \mathrm{~min}$. The pellet and supernatant fractions were analyzed by SDS-PAGE, followed by Coomassie blue staining and autoradiography.

\section{Clathrin assembly assay}

Three molar solution of clathrin triskelia were dialyzed overnight at $4^{\circ} \mathrm{C}$ against isolation buffer with the addition of assembly protein ([ $\left[{ }^{35} \mathrm{~S}\right]$-methionine labeled CALM protein, reticulocyte lysate). Following centrifugation at $13,600 \mathrm{~g}$ for $3 \mathrm{~min}$ to remove nonspecific aggregates, newly assembled clathrin cages were precipitated by ultracentrifugation for 20 minutes at $100,000 \mathrm{~g}$. The pellet and the supernatant fractions were analysed by SDS-PAGE, followed by Coomassie blue staining and autoradiography.

\section{SH3 (Src homology 3) domain binding assay}

GST-SH3 domain proteins and GST proteins were expressed in $\mathrm{E}$. coli and immobilized on glutathion sepharose $4 \mathrm{~B}$ beads. Then the beads were incubated for $2 \mathrm{~h}$ at $4^{\circ} \mathrm{C}$ with $5 \mu \mathrm{l}$ of CALM protein in $500 \mu \mathrm{l}$ of the binding buffer (20 mM Tris, $\mathrm{pH} 7.5,150 \mathrm{mM} \mathrm{NaCl}, 0.5 \%$ NP 40). Sample was centrifuged for $10 \mathrm{~min}$ at $10,000 \mathrm{~g}$ at $4^{\circ} \mathrm{C}$. The supernatant was carefully aspirated and the beads were washed with $500 \mu \mathrm{l}$ of binding buffer. And the samples were centrifuged for $10 \mathrm{~min}$, at 10,000 $\mathrm{g}$ at $4^{\circ} \mathrm{C}$. The samples were washed the same way four times. Bound protein was eluted by boiling in standard SDS-gel loading buffer, separated by $10 \%$ SDS-PAGE and visualized by autoradiography.

\section{Dephosphorylation of the CALM protein}

Five microliters of the labelled CALM were mixed with 40 unit of calf intestine alkaline phosphatase in reaction buffer (50 mM Tris, pH 9.0, $1 \mathrm{mM} \mathrm{MgCl}_{2}$ ) or mixed with only reaction buffer as negative control. The mixtures were incubated for $1 \mathrm{~h}$ at $30^{\circ} \mathrm{C}$. The change in mobility was analyzed by SDS-PAGE followed by autoradio- 
graphy.

\section{Proteolysis of CALM}

The labelled CALM protein $(5 \mu \mathrm{l})$ was mixed with lysate containing caspase in $20 \mu \mathrm{l}$ of reaction buffer $(16 \mathrm{mM}$ HEPES, $8 \mathrm{mM} \mathrm{NaCl}, 0.004 \%$ IGEPAL-CA630). The mixtures were incubated for $1 \mathrm{~h}$ at $30^{\circ} \mathrm{C}$. The CALM protein $(5 \mu \mathrm{l})$ was mixed with calpain (Sigma) in $20 \mu \mathrm{l}$ of reaction buffer (1X PBS, $1 \mathrm{mM} \mathrm{CaCl} 2$ ). The mixtures were incubated for $1 \mathrm{~h}$ at $37^{\circ} \mathrm{C}$. The digestions of CALM were analyzed by SDS-PAGE followed by autoradiography.

\section{Results and Discussion}

\section{Expresseion of CALM and preperation of clathrin, and AP180}

We expressed the CALM using cell-free reticulocyte lysate translation system. SDS-PAGE of the [ $\left.{ }^{35} \mathrm{~S}\right]-$ methionine labeled proteins revealed a major band with an apparent molecular weight of $105 \mathrm{kD}$ (Figure 1A). This is consistent with the previous report from rat liver (Kim et al., 2000a), that produced native CALM with an apparent MW of $110 \mathrm{kD}$ on SDS-PAGE. The molecular weight is however somewhat larger than that estimated $71 \mathrm{kD}$ mass weight based on amino acid composition. The discrepancy in the MW between native and the estimated size was also observed in AP180. The unusual migration of CALM in PAGE was likely caused by high ratio of proline, glycine and charged amino acid. The CALM contains $23 \%$ of charged amino acids, and $14 \%$ of proline residues (Kim et al., 1999). The posttran-

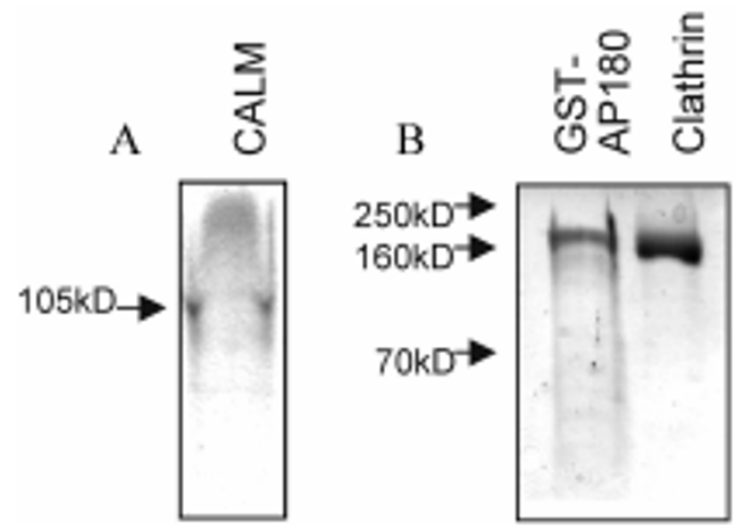

Figure 1. SDS-PAGE analysis of the $\left[{ }^{35} \mathrm{~S}\right]$-methionine labeled CALM protein and the purified proteins. $\left[{ }^{35}\right.$ S]-methionine labeled CALM was expressed in cell-free rabbit reticulocyte system by in vitro translation. GSTAP180 was purified from extracts of $E$. coli BL21 expressing the recombinant protein. Clathrin was purified from bovine brain - coated vesicle. The in vitro translated $\left[{ }^{35} \mathrm{~S}\right]-$ methionine labeled CALM was analyzed by SDS-PAGE followed by autoradiography (A) and GST-AP180 and clathrin are analyzed by SDS-PAGE followed by Coomassie blue staining $(B)$. slational modification could also affect the eletrophoretic mobility in the gel. These might result in anomalous migration of the CALM on SDS-PAGE, with differences between true and apparent molecular weights. The purity of the AP180 and clathrin used in the experiment were shown in Figure 1B. AP180 was prepared by using GST (Glutathione-S-transferase) expression system, and the staining patterns of Coomassie blue and immunoblotting by anti-AP180 monoclonal antibody were identical. Clathrin heavy chain from bovine brain was identified as single band on SDS-PAGE, and the light chains were not shown on the gel condition.

\section{Binding of the CALM protein with preassembled clathrin cage and clathrin assembly}

The binding ability of CALM to clathrin cages was monitored by the cosedimentation of CALM and clathrin cages. AP180 (a positive control) were cosedimented with preassembled clathrin cages in the pellet fraction after ultracentrifugation at $100,000 \mathrm{~g}$, in the presence of clathrin cages (Figure 2A, lane 4). CALM dialyzed with clathrin cages, sedimented with the clathrin cages by the $100,000 \mathrm{~g}$ centrifugation but in the absence of clathrin cages (Figure $2 \mathrm{~A}$ and $2 \mathrm{~B}$, lane $5,6,7$, and 8 ) indicating that CALM was able to bind the preassembled clathrin cages. The ability of clathrin cage assembly of CALM was monitored by dialysis with clathrin triskelia and the CALM protein, and the clathrin cage formed from the CALM and clathrin triskelia could be seperated by $100,000 \mathrm{~g}$ centrifugation on the ultracentrifuge. We used AP180, as a positive control in assembly assay. The cosedimention of CALM protein with clathrin was detect-

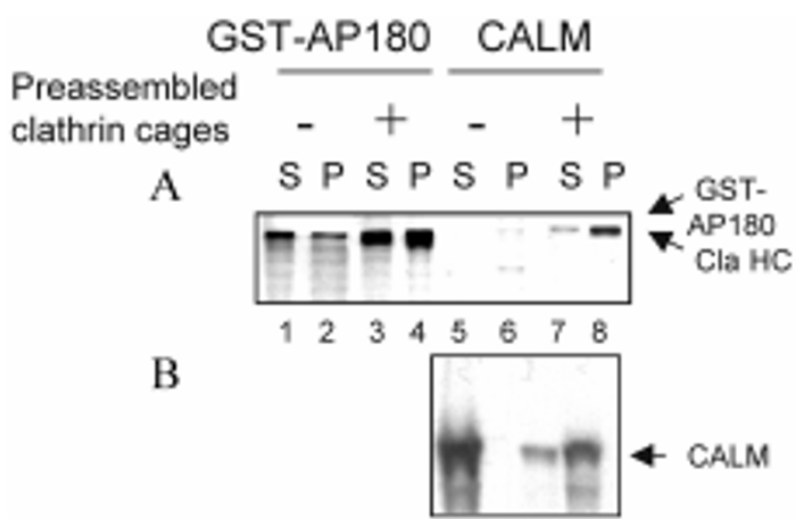

Figure 2. Binding of preassembled cage by the CALM protein. Clathrin cage binding assays were carried on as described in "Materials and Methods". Twenty $\mu \mathrm{M}$ concentration of GST-AP180 (lane 1-4), $5 \mu$ of labeled CALM in reticulocyte lysate (lane 5-8) were incubated in the absence $(-)$ or presence $(+)$ of $1.8 \mu \mathrm{g}$ (corresponding to molarity of triskelion, $1.2 \mathrm{mg} / \mathrm{ml}$ ) preassembled clathrin cage. Following a low spin to remove nonspecific aggregates, all samples were pelleted by ultracentrifugation at $100,000 \mathrm{~g}$. The supernatant $(\mathrm{S})$ and the pellet $(\mathrm{P})$ fractions were analyzed by SDS-PAGE, followed by Coomassie blue staining (upper panel) and autoradiography (lower pannel). Positions of each protein are indicated by clathrin heavy chain (Cla HC), GST-AP180, CALM. 


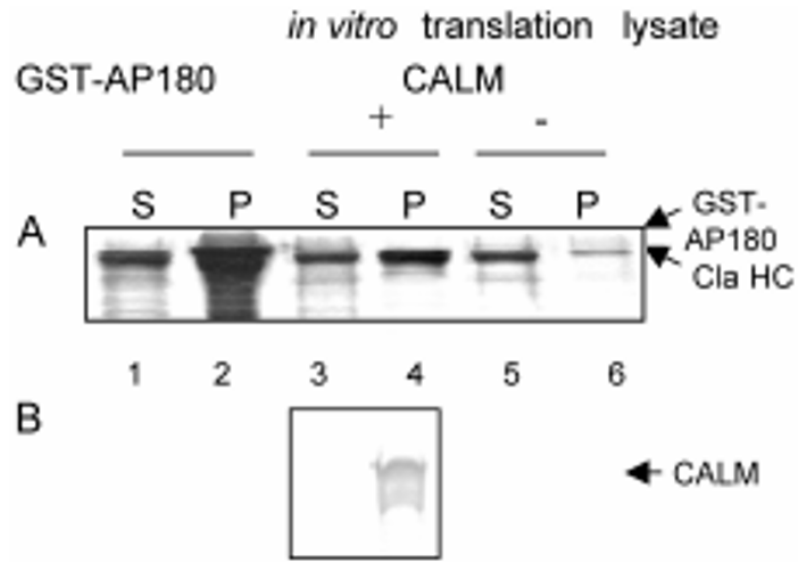

Figure 3. Assembly of clathrin cage by the CALM protein. Clathrin assembly assays were performed as described in "Materials and Methods". $3 \mu \mathrm{M}$ clathrin triskelia were dialyzed overnight at $4^{\circ} \mathrm{C}$ against isolation buffer with the addition of $20 \mu \mathrm{M}$ concentrations of GST-AP180, $10 \%$ of the translated $\left[{ }^{35} \mathrm{~S}\right]$-methionine labeled CALM in reticulocyte lysate. Following a low spin to remove nonspecific aggregates, newly assembled clathrin cages were pelleted by ultracentrifugation at $100,000 \mathrm{~g}$. The supernatant (S) and the pellet $(\mathrm{P})$ fractions were analyzed by $10 \%$ SDS-PAGE, followed by Coomassie blue staining (upper panel) and autoradiography (lower panel). Positions of each protein are indicated in the same way as in Figure 2.

ed by it's radioactivity in 100,000 $\mathrm{g}$ pellet fraction (Figure 3B, lane 4). Protein staining of the gel also showed a significant increase of clathrin proteins in the $100,000 \mathrm{~g}$ pellet fraction (Figure $3 A$, lane 4) demonstrating the ability of CALM to promote clathrin triskelia into cages.

CALM isolated from liver was reported to bind with clathrin heavy chain (Tebar et al., 1999) and could assemble clathrin triskelia into cages (Kim et al., 2000b). Moreover, the observation that bacterially expressed CALM could bind to preassembled clathrin cage and assemble clathrin cage (Kim et al., 2000b) suggests that on a gross appearance, the posttranslational modification of the CALM may not significantly alter the clathrin binding activity and cage-formation activity of the CALM although a kinetic profile of binding activities of CALM via cell signal-induced phosphorylation cannot be ruled out with these data. The CALM protein has more than $95 \%$ homology with AP180 in N terminus, but the sequence of $\mathrm{C}$ terminal region is different from that of AP180 (Dreyling et al., 1996; Kim et al., 1999). Apparently, both $\mathrm{N}$ terminal $(33 \mathrm{kD})$ and $\mathrm{C}$ terminal $(55 \mathrm{kD})$ parts of the AP180 have clathrin-binding activity (Ye et al., 1995) and C terminal domain has the activity of clathrin cage assembly. Vesicle formation could be regulated through binding of inositol phosphate, other adaptor protein, and phosphorylation of assembly protein. AP180 has high affinity to inositol hexakisphosphate and diphosphoinositol pentakisphosphate. The CALM protein possesses inositol binding motif and able to bind hexakisphosphate (Kim et al., 1999). Binding of these phosphoinositides inhibits clathrin assembly (Norris et al., 1995). Because of similar molecular structure between

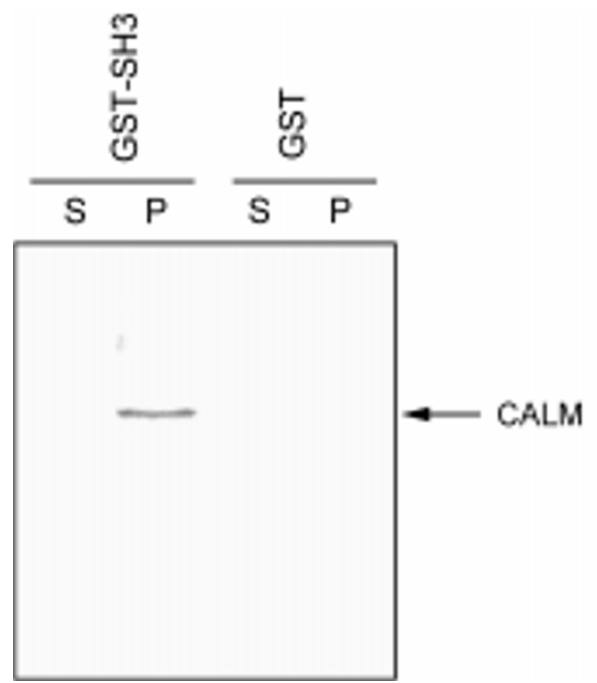

Figure 4. Interaction of the CALM protein with $\mathrm{SH} 3$ domain. SH3 binding assays were performed as described in "Materials and Methods". The proteins (GST-SH3, GST) bound to the beads and $5 \mu$ of labeled CALM were used in the binding assay. The supernatant $(S)$ and the pellet $(P)$ fractions were analyzed by $10 \%$ SDS-PAGE followed by autoradiography.

CALM and AP180, the formation of CCV by CALM might also be regulated by the phosphoinositides.

\section{The interaction of the CALM protein with $\mathrm{SH} 3$ domain}

CALM has multiple proline rich regions in the $C$ terminal, which would interact with both $\mathrm{SH} 3$ and WW domain. Furthermore, it has some PxxP motif, which is a known ligand for $\mathrm{SH} 3$ domain. To confirm the direct interaction between CALM and $\mathrm{SH} 3$ domain through the proline rich region, the GST-pull down assay was carried out in vitro. In this assay, GST-PKC $\gamma$ (protein kinase $\mathrm{C}$ ) $\mathrm{SH} 3$ domain - immobilized agarose beads were incubated with CALM and the bound protein was analyzed by SDSPAGE followed by autoradiography. The result showed that CALM did interact with SH3 domain (Figure 4, lanes 1 and 2). It failed to interact with a fusion partner GST alone (Figure 4, lanes 3 and 4). The clathrin vesicle associated proteins harboring $\mathrm{SH} 3$ domain are dynamin and the amphiphysin. Dynamin is a cytosolic guanosine triphosphatase (GTPase), which has a role of pinching off the clathrin coated pit. Amphiphysin has a $\mathrm{SH} 3$ domain, which binds the GTPase dynamin, the inositol5-phophatase, synaptojanin-1 and tetrameric clathrin assembly protein AP-2 (Zhang et al., 1994; Cestra et al., 1999).

\section{Dephosphorylation of the CALM protein by alkaline phosphatase}

AP180, a CALM homologous protein, was known to be a phosphoprotein and to be phosphorylated by casein kinase II (Hao et al., 1999). CALM has eight possible phosphorylation sites (Kim et al., 1999). The major sites 


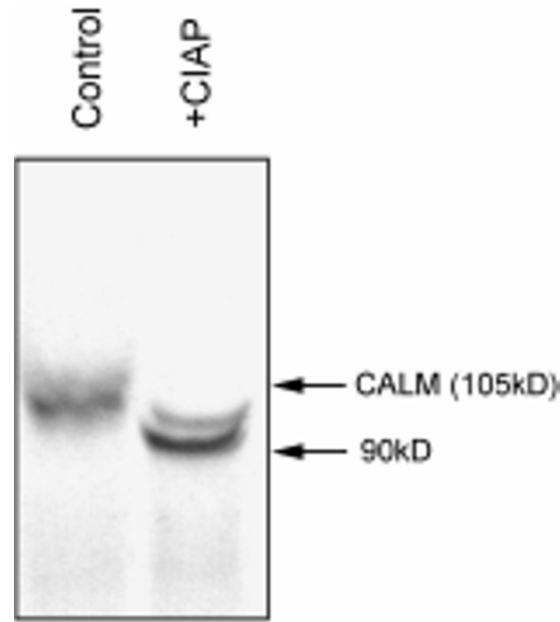

Figure 5. Dephosphorylation of the CALM protein by alkaline phosphatase. Five $\mu \mathrm{l}$ of the labeled CALM in reticulocyte lysate was treated with only calf intestine alkaline phsphatase buffer as a negative control (lane 1), and 4 unit of calf intestine alkaline phosphatase (CIAP) and reaction buffer (lane 2). Reactions were analyzed by $10 \%$ SDS-PAGE, followed by autoradiography.

of phophorylation are located on the $\mathrm{N}$ terminal. In an effort to test whether the expressed CALM, by reticulocyte lysate translation system, was phosphorylated, it was treated with calf intestine alkaline phosphatase and analyzed by SDS-PAGE for any change in the electrophoretic mobility. Indeed, alkaline phosphatase treatment led to a distinct increase of the elctrophoretic mobility of CALM band as well as an increase in its sharpness (Figure 5), corresponding to a decrease in the apparent molecular size of CALM protein from 105 $\mathrm{kD}$ to $90 \mathrm{kD}$ but since the electrophoretic mobility of CALM is anomalous, the decrease in molecular size can not be correlated to the number of phosphorus groups. However, the result definitively supports that CALM expressed in the cell-free reticulocyte lysate system is phosphorylated. Phosphorylation of AP180 was reported to decrease its interaction with AP-2. The assembly activity of the combination of phosphorylated AP180 and AP-2 was affected due to its reduced affinity for AP-2 (Hao et al., 1999). Phosphorylation mediated modulation of CALM interaction with other adaptor protein is a strong possibility. In turn, CALM may participate in and be regulated in the formation of CCVs, by its phosphorylation state or interacting with $\mathrm{SH} 3$-harboring protein.

\section{Proteolysis of the CALM protein by caspase 3 and calpain}

The CALM has 3 DxxD motif at amino acids 263, 392, and 526 from amino terminus. DxxD motif could be the substrate of caspase 3 . To confirm the proteolysis by caspase, CALM was treated with caspase 3 and 8. Protealysis of CALM by caspase 3 was evident, but not by caspase 8 (Figure 6, lanes 2 and 3). Caspase 3, an

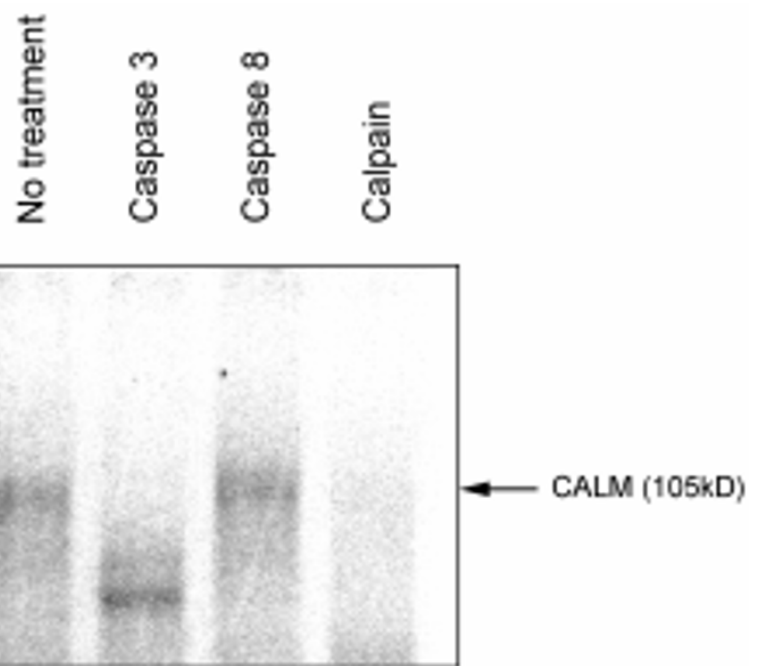

Figure 6. Proteolysis of the CALM protein by caspase 3 and calpain. Five $\mu \mathrm{l}$ of the labeled CALM in reticulocyte lysate was treated with caspase 3 (lane 2), caspase 8 (lane 3), and calpain (lane 4), and no treatment as a negative control (lane 1). Reactions were analyzed by $10 \%$ SDS-PAGE followed by autoradiography.

effector caspase, was activated by caspase 8 or caspase 9 by different pathways (Nunez et al., 1998). Caspases are known to be activated during only apoptosis cascade. Apoptotic proteolysis of vesicle-associated proteins by caspases were not reported to date and it is the first case of CALM being a substrate for caspase. To investigate whether the CALM can be cleaved by calpain, a $\mathrm{Ca}^{2+}$-dependent protease, the radiolabeled CALM was digested with calpain. The intensity of the CALM band was markedly reduced without showing any laddering fragmentation pattern (Figure 6, lane 4), suggesting the presence of possible multiple calpain-cleavage sites in CALM. The calpain sensitivity of CALM may provide mechanism through which its activity is regulated by changing intracellular calcium concentration. The proposed involvement of caspase and calpain in the processes of the neurodegeneration by apoptosis may imply a role for the CALM. The CALM protein, known to possess some protein binding motifs and distributed widely in various tissues, may play a role as a clathrin assembly protein as well as an adaptor protein (Marsh et al., 1999). Our findings with CALM protein expressed in the controlled eukaryotic system showed it's participation in the formation of the clathrin vesicle and raised strong possibility of its involvement in modulation of interacting with other proteins harboring $\mathrm{SH} 3$ domain, through its phosphorylation, and by proteolytic cleavage.

\section{Acknowledgement}

This study was supported by the Grant from the Korea Science and Engineering foundation (981-074-032-2), 
Korea, 1998, awarded to Hyung-Lae Kim.

\section{References}

Ahle S., Ungewickell E. Purification and properties of a new clathrin assembly protein. EMBO J. 1986;5:3143-49

Alberts B, Bray D, Lewis J, Raff M, Roberts K, Watson J. Molecular Biology of The Cell. 3rd ed., 1994, pp. 634-46. Garland Pub. New York.

Bar-Zvi D, Mosley ST, Branton D. (1988) In vivo phosphorylation of clathrin-coated vesicle proteins from rat reticulocytes. J Biol Chem 1988;263:4408-15

Beck KA, Chang M, Brodsky FM, Keen JH. Clathrin assembly protein AP-2 induces aggregation of membrane vesicles: a possible role for AP-2 in endosome formation. J Cell Biol 1992;119:787-96

Cestra G, Castagnoli L, Dente L, Minenkova O, Petrelli A, Migone N, Hoffmuller U, Schneider-Mergener J, Cesareni G. The SH3 domains of endophilin and amphiphysin bind to the proline-rich region of synaptojanin 1 at distinct sites that display an unconventional binding specificity. J Biol Chem 1999;274:32001-7

Dell'Angelica EC, Mullins C, Bonifacino JS. AP-4, a novel protein complex related to clathrin adaptors. J Biol Chem 1999;274:7278-85

Dreyling $\mathrm{MH}$, Martinez-Climent JA, Zheng M, Mao J, Rowley JD, Bohlander SK. The $t(10 ; 11)(p 13 ; q 14)$ in the U937 cell line results in the fusion of the AF10 gene and CALM, encoding a new member of the AP-3 clathrin assembly protein family. Proc Natl Acad Sci USA. 1996;93:4804-9

Faundez V, Horng JT, Kelly RB. A function for the AP3 coat complex in synaptic vesicle formation from endosomes. Cell 1998;93:423-32

Hao W, Luo Z, Zheng L, Prasad K, Lafer EM. AP180 and AP2 interact directly in a complex that cooperatively assembles clathrin. J Biol Chem 1999;274:22785-94

Keen JH, Black MB. The phosphorylation of coated membrane proteins in intact neurons. J Cell Biol 1986;102:1325-33

Kim HL, Lee $\mathrm{CH}$. Molecular cloning of clathrin assembly protein gene (rCALM) and its differential expression to AP180 in rat brain. Exp Mol Med 1999;31:165-69

Kim HL, Kim JA. Purification of clathrin assembly protein from rat liver. Exp Mol Med 2000a; 32:222-26

Kim JA, Kim SR, Jung YK, Woo SY, Seoh JY, Hong YS, Kim HL. Properties of GST-CALM expressed in E. coli. Exp Mol Med 2000b;32:93-99

Marsh M, McMahon HT. The structural era of endocytosis. Science 1999;285:215-20

Morris SA, Schroder S, Plessmann U, Weber K, Ungewickell E. Clathrin assembly protein AP180: primary structure, domain organization and identification of a clathrin binding site. EMBO J. 1993;12:667-75

Murphy JE, Pleasure IT, Puszkin S, Prasad K, Keen JH. Clathrin assembly protein AP-3. The identity of the $155 \mathrm{~K}$ protein, AP180, and NP185 and demonstration of a clathrin binding domain. J Biol Chem 1991;266:4401-8

Norris FA, Ungewickell E, Majerus PW. Inositol hexakisphosphate binds to clathrin assembly protein 3(AP-3/AP180) and inhibits clathrin cage assembly in vitro. J Biol Chem 1995;270: 214-17

Nunez G, Benedict MA, Hu Y, Inohara N. Caspase: the protease of the apoptotic pathway. Oncogene 1998;17:323745

Simpson F, Peden AA, Christopoulou L, Robinson MS. Characterization of the adaptor-related protein complex, AP-3. J Cell Biol 1997;137:835-45

Stephan AM, Annette M, Ungewickell E. Anaysis of 100-180 $\mathrm{kD}$ phosphoprotein in clathrin-coated vesicles from bovine brain. J Biol Chem 1990;265:3354-57

Tebar F, Bohlander SK, Sorkin A. Clathrin assembly lymphoid myeloid leukemia (CALM) protein: localization in endocyticcoated pits, interactions with clathrin, and the impact of overexpression on clathrin-mediated traffic. Mol Biol Cell 1999;10: 2687-702

Ye W, Lafer EM. Clathrin binding and assembly activities of expressed domains of the synapse-specific clathrin assembly protein AP-3. J Biol Chem 1995;270:10933-37

Zhang JZ, Davletov BA, Sudhof TC, Anderson RGW. Synaptotagmin I is high affinity receptor for clathrin AP-2: implications for membrane recycling. Cell 1994;78:751-60 